\title{
Renal Complications after Hematopoietic Stem Cell Transplantation: Role of Graft-Versus-Host Disease in Renal Thrombotic Microangiopathy
}

\author{
Akiko Mii $^{1}$, Akira Shimizu ${ }^{2}$, Hiroki Yamaguchi ${ }^{3}$ and Shuichi Tsuruoka ${ }^{1}$ \\ ${ }^{1}$ Department of Nephrology, Nippon Medical School, Tokyo, Japan \\ ${ }^{2}$ Department of Analytic Human Pathology, Nippon Medical School, Tokyo, Japan \\ ${ }^{3}$ Department of Hematology, Nippon Medical School, Tokyo, Japan
}

\begin{abstract}
Hematopoietic stem cell transplantation (HSCT) is a widely accepted treatment for mainly hematopoietic disorders. Recent advances in transplantation techniques have increased the number of long-time survivors with chronic kidney disease (CKD). The presence of CKD affects outcomes and is associated with high mortality rates. Therefore, physicians treating transplant survivors should consider renal complications and optimize management of patients with CKD after HSCT. The pathology of CKD after HSCT is affected by many factors, and the causes of renal thrombotic microangiopathy (TMA) are diverse and complicated. We have treated patients who underwent allogeneic HSCT and developed latestage renal TMA possibly associated with graft-versus-host disease (GVHD). Administration of immunosuppressive drugs, such as calcineurin inhibitors, is typically reduced in patients with TMA. However, if renal TMA is caused by renal GVHD, the use of immunosuppressive drugs should be increased, contradicting conventional thinking. On the basis of previous findings and our own observations, we review the pathology of renal complications after HSCT and focus on the role of GVHD in the development of renal TMA. (J Nippon Med Sch 2020; 87: 7-12)
\end{abstract}

Key words: chronic kidney disease, hematopoietic stem cell transplantation, thrombotic microangiopathy, graft-versus-host disease

\section{Introduction}

An increasing number of patients are undergoing hematopoietic stem cell transplantation (HSCT) to treat hematopoietic disorders such as leukemia and malignant lymphoma. Worldwide, more than 60,000 patients undergo HSCT annually. In Japan, more than 5,000 patients (allogeneic: 3,500 and autologous: 1,500 ) a year undergo HSCT, and more than 70,000 patients have been treated with the technique during the past 20 years ${ }^{2}$. Cases of long-term survival are increasing with improvements in transplantation techniques; however, renal disorders that complicate the late stages after transplantation often progress to end-stage renal failure, which may lead to renal replacement therapy ${ }^{3,4}$. Furthermore, renal complications increase morbidity and mortality rates ${ }^{5}$.

Medical professionals involved in transplantation medicine, including hematologists and nephrologists, should understand the pathology of renal disorders associated with HSCT, including the relevant treatment perspectives. However, the pathology of renal complications after HSCT is complicated, so clarifying the disease characteristics and establishing appropriate therapeutic methods are important yet difficult tasks.

We recently reported that renal thrombotic microangiopathy (TMA) is associated with chronic graft-versushost disease (GVHD) ${ }^{6-8}$. Transplant-associated TMA (TATMA) is generally diagnosed on the basis of laboratory findings such as hemolytic anemia, thrombocytopenia, and elevated lactate dehydrogenase level, because it is difficult to obtain tissue specimens from patients with severe physical conditions. In contrast, renal TMA refers to pathological TMA in the kidney, with or without sys-

Correspondence to Akiko Mii, MD, PhD, Department of Nephrology, Nippon Medical School, 1-1-5 Sendagi, Bunkyo-ku, To-

kyo 113-8603, Japan

E-mail: m-akiko@nms.ac.jp

https://doi.org/10.1272/jnms.JNMS.2020_87-102

Journal Website (https://www.nms.ac.jp/sh/jnms/) 
temic symptoms 9 . Characteristic morphological findings in renal TMA include fibrin thrombi within glomeruli and mesangiolysis and/or thrombosis involving arterioles or interlobular arteries, in conjunction with characteristic intimal swelling in vessels and occlusion of capillary loops ${ }^{7,9}$. We have treated patients with renal TMA in which GVHD may have been involved in the pathogenesis. Renal GVHD has not been defined, although nephrotic syndrome is a well-known renal manifestation in chronic GVHD. In addition, the association between renal TMA and renal GVHD is controversial. In this article, we describe renal complications after HSCT, particularly the association between renal TMA and renal GVHD, and review the literature and our own research.

\section{Review of Previous Literature}

\section{Renal Complications after HSCT}

In the early stage after transplantation, the incidence of acute kidney injury (AKI) caused by renal complications associated with HSCT is high, and the incidence rate of AKI is higher after allogeneic HSCT than after autologous $\mathrm{HSCT}^{10,11}$. The causes of AKI are believed to be severe infection, nephrotoxic drugs, acute GVHD, and systemic TMA in the presence of schistocytes. In contrast, during the late stage after transplantation, renal function gradually deteriorates for various reasons and ultimately progresses to end-stage renal failure ${ }^{3}$. The causes of chronic kidney disease (CKD) are repeated AKI attributable to various causes, long-term use of calcineurin inhibitors (CNIs) for GVHD prophylaxis and treatment, radiation nephropathy, nephrotic syndrome caused by GVHD, and renal TMA with or without systemic symptoms $s^{4,12}$. In general, health-related quality of life is substantially lower for people with CKD than for the general population. CKD is associated with cardiovascular risks and increased incidences of several cancers ${ }^{13,14}$. Similarly, post-HSCT patients with CKD have a lower overall survival rate than those without $C K D^{15}$. Previous studies of CKD after HSCT suggest that the pathology of renal disorders associated with transplantation is complicated and multifactorial. Careful monitoring of renal function, including albuminuria, is required for long-term survi$\operatorname{vors}^{16,17}$

\section{Renal Complications Associated with GVHD}

Among the renal complications associated with GVHD, nephrotic syndrome is well known but rare ${ }^{18,19}$. Most kidney biopsy images show membranous nephropathy with subepithelial deposits. Therefore, antigen-antibody complexes due to GVHD may be present. In addition, renal histopathological findings may include minimal change disease, focal segmental glomerulosclerosis, and IgA nephropathy ${ }^{20,21}$. Increased production of tumor necrosis factor-alfa (TNF- $\alpha$ ) and interferon-gamma (IFN- $\gamma$ ) by donor $\mathrm{T}$ cells was associated with development of nephrotic syndrome ${ }^{22}$. Disease activity increased after a dose reduction in immunosuppressive drugs, and increased drug administration improved proteinuria, which suggests that the symptoms were caused by GVHD. In contrast, an examination of autopsy cases by Kusumi et al. suggested that peritubular capillaritis (PTC-itis) and tubulitis may be GVHD-related lesions ${ }^{23}$. We also believe these findings to be related to renal GVHD. Kusumi et al. further speculated that the Banff classification for kidney transplantation may be a useful tool to assess renal pathology after allogeneic HSCT. The precise mechanism underlying kidney injury and GVHD is unclear, although the kidney is believed to be a target organ of GVHD.

\section{Vascular Injury Mediated by GVHD}

GVHD is an immune-mediated disorder believed to be driven by allospecific lymphocytes, as it does not occur after autologous HSCT. GVHD develops when donorderived $\mathrm{T}$ cells respond to recipient tissues. There are two important principles when considering the pathophysiology of acute GVHD. First, acute GVHD is a severe but normal inflammatory response mediated by donor lymphocytes infused into the recipient. Second, recipient tissues that stimulate donor lymphocytes are usually vulnerable because they have been damaged by the underlying disease, chemotherapy, prior infection, and/ or the HSCT conditioning regimen. As a result, these tissues produce inflammatory cytokines that promote activation and proliferation of donor immune cells ${ }^{24}$. Chronic GVHD is also an important cause of morbidity and mortality in long-term survivors of allogeneic HSCT.

The pathophysiology of chronic GVHD is more complicated. Recent studies of chronic GVHD have increasingly focused on the importance of B cells, while earlier studies focused on $\mathrm{T}$ cells. Humoral reaction is mediated by altered B cell subpopulations, danger B cell signaling pathways, autoantibodies, and T cell-B cell interactions ${ }^{25}$. In general, GVHD involves epithelial tissues of the skin, gastrointestinal tract, and lungs. GVHD can also damage endothelial cells, and transplant-associated microangiopathy (TA-TMA) may represent GVHD. Plasma levels of markers of endothelial cell injury and the coagulation system are elevated in patients with acute GVHD after HSCT, suggesting an association of endothelial injury, acute GVHD, and subsequent development of $\mathrm{TMA}^{26-28}$. 
Increasing evidence indicates that endothelial injuries mediated by GVHD are present. In the early stages after HSCT, severe endothelial injuries, such as TA-TMA, veno-occlusive disease of the liver, diffuse alveolar hemorrhage, engraftment syndrome, and capillary leak syndrome, all share features with acute $\mathrm{GVHD}^{29}$. In late stages, persistent endothelial injury associated with chronic GVHD leads to microvascular rarefaction, loss of microvessels, and, ultimately, to organ fibrosis. It is unclear whether the main contributor mediating endothelial cell injury is the $\mathrm{T}$ cell, another cell type, or another mechanism ${ }^{28}$. Furthermore, late cardiovascular accidents are more likely to occur in patients treated with allogeneic HSCT than in those treated with autologous HSCT, which suggests that an immunological mechanism is involved in atherosclerosis development. In solid-organ transplants, vascular endothelial cells are an important target for alloreactive cytotoxic $\mathrm{T}$ cells in vascular rejection $^{30}$. Endothelialitis, intimal arteritis, and subendothelial infiltration of the arterial intima by cytotoxic $\mathrm{T}$ cells are hallmark histopathological lesions of severe acute rejection of transplants. Chronic graft rejection, characterized by replacement fibrosis of graft parenchyma, is a frequent consequence of tissue ischemia resulting from progressive loss of microvessels. The mechanism underlying vascular injury associated with GVHD in HSCT patients may be similar to that associated with vascular rejection in solid-organ transplant patients. In fact, after allogeneic HSCT, vascular endothelial cells are the first allogeneic recipient cells encountered by circulating immunocompetent donor $\mathrm{T}$ cells. In human studies, vascular injury has been reported in patients with acute GVHD, and arterial changes similar to allograft vasculopathy have been reported in patients with chronic GVHD ${ }^{28}$.

\section{TA-TMA and Pathological Renal TMA}

Several diagnostic criteria for TA-TMA that reflect clinical findings have been proposed, namely, (1) hemolytic anemia, (2) thrombocytopenia, (3) elevated lactate dehydrogenase level, and (4) presence of schistocytes in peripheral blood or a blood smear. Jodele et al. ${ }^{31}$ proposed that if any of these findings are observed during examination or if TMA findings are observed in the tissue, then TA-TMA should be diagnosed. In addition, elevated circulating C5b-9 (a marker of terminal complement activation), elevated blood pressure, and proteinuria may be indicators of TA-TMA. In a study of autopsy cases after HSCT, many exhibited clinical and pathological TMA findings ${ }^{32}$. Some cases of non-clinical TMA also showed evidence of pathological renal TMA. Even when systemic symptoms are absent, the kidney may exhibit TMA with severe endothelial cell injury. Changsirikulchai et al. ${ }^{9}$ examined cases exhibiting TMA "histologically", regardless of whether the diagnosis was clinical or nonclinical TMA. Light microscopic observations of pathological renal TMA showed fibrin thrombi within glomeruli, mesangiolysis and duplication of glomerular basement membrane, and/or thrombus involving arterioles or interlobular arteries, regardless of the clinical characteristics of TMA. With or without clinical TMA manifestations, these findings suggest that acute GVHD is a risk factor for renal TMA, but the use of CNI was not affected. The authors recommended that CNI should not be reduced or stopped because of renal TMA. Recently, another group reported that complement dysregulation may be involved in TA-TMA pathogenesis. Elevated soluble $\mathrm{C} 5 \mathrm{~b}-9$ and total complement activity (CH50) were observed in TA-TMA cases. Furthermore, eculizumab (a C5 inhibitor) may be useful for treating TA-TMA ${ }^{33}$.

The causes of renal TMA are diverse. In general, TMA is believed to be caused by use of CNIs for GVHD prophylaxis, viral infection, and total body irradiation. Therefore, administration of immunosuppressive drugs is commonly reduced or stopped. However, while this is common, we consider that renal TMA after allogeneic HSCT might result from GVHD and that administration of immunosuppressive drugs should thus be increased.

\section{Authors' Findings \\ Renal TMA after Allogeneic HSCT: Involvement of Chronic GVHD}

We reported a case of progressive renal insufficiency accompanied by proteinuria 2 years after umbilical cord blood transplantation for refractory myelodysplastic syndrome $^{6}$. A kidney biopsy showed severe endothelial cell injury of microvessels. Renal TMA was diagnosed, even though there were no clinical symptoms of TMA, except for hypertension (Fig. 1) ${ }^{6}$. We initially thought that kidney injury was caused by use of CNI for GVHD prophylaxis. However, renal function deteriorated when CNI was gradually reduced and stopped. The patient ultimately required dialysis.

We were unsure of the cause of renal impairment. Further pathological analysis showed that, in addition to vascular endothelial cell injury, there was glomerulitis, PTC-itis, tubulitis, and C4d deposition in the glomerular basement membrane and patchy PTCs. These findings were similar to those seen in images of chronic antibodymediated rejection in kidney transplantation. These find- 


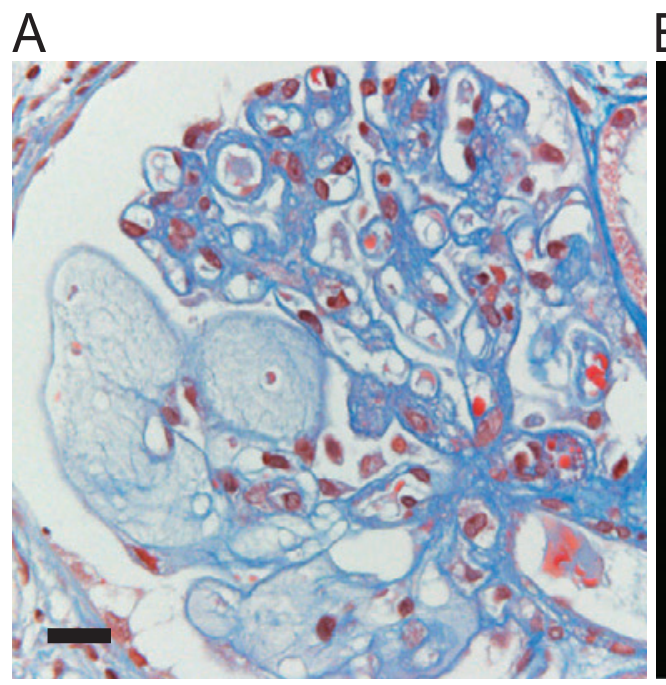

B

Fig. 1 Renal TMA characterized by severe endothelial injury. (A, Masson trichrome stain; B, C4d stain; scale bar $=50 \mu \mathrm{m}$ )

Representative images of renal TMA characterized by diffuse global enlarged subendothelial spaces, double contouring of the glomerular basement membrane, and post-mesangiolysis lesions (A). A diffuse, global pattern of C4d deposition was detected on glomerular capillaries. Patchy staining for C4d was also evident in peritubular capillaries (C). Reprinted and modified from Pathology International, 61, Mii A et al., Renal thrombotic microangiopathy associated with chronic humoral graft versus host disease after hematopoietic stem cell transplantation, 34-41, Copyright (2011), with permission from Wiley.

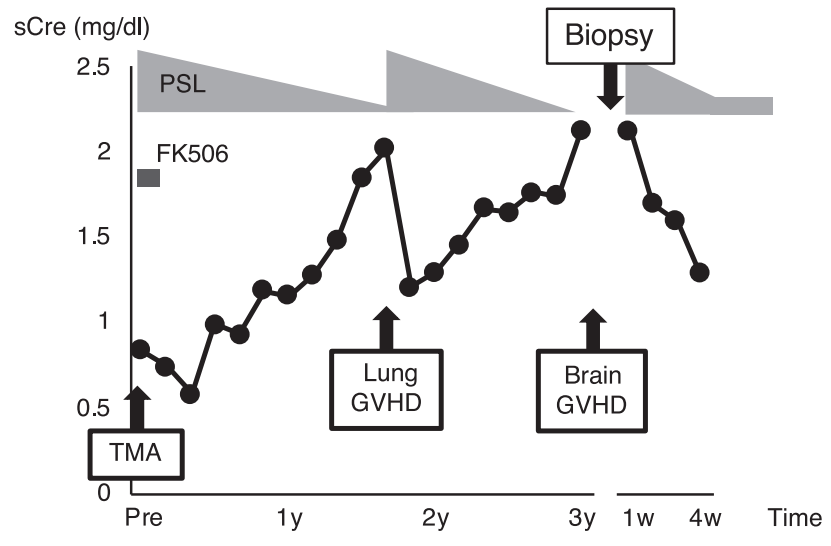

Fig. 2 Time course of serum creatinine levels during treatment for renal GVHD with renal TMA.

This figure shows the clinical course of a representative case. Prednisolone was used mainly for GVHD treatment after umbilical cord blood transplantation (UCBT). When prednisolone dose was reduced, lung GVHD and brain GVHD developed along with progressive renal insufficiency. An increase in prednisolone dose led to improvement in physical findings and renal function. Thus, renal insufficiency and involvement of other organs might be linked. Reprinted from Kidney International Reports, 3, Mii A et al., Renal Thrombotic Microangiopathy After Hematopoietic Stem Cell Transplantation: Involvement of Chronic GraftVersus-Host Disease. 743-747, Copyright (2018), with permission from Elsevier. ings suggested that the cause of renal TMA in this case might be chronic renal GVHD, in which chronic humoral GVHD may have caused antibodies to renal microvascular endothelial cells.

Later, two biopsy and five autopsy cases were investigated $^{7}$. In all cases, pathological findings showed widespread, persistent endothelial cell injury in glomeruli, PTCs, and small arteries, indicating pathological renal TMA, although five of the seven cases did not satisfy the clinical criteria for systemic TMA. Furthermore, we identified glomerulitis, tubulitis, and PTC-it is, together with activated cytotoxic $\mathrm{T}$ cells, in all cases. Tubulitis and PTC-itis are characteristic findings of GVHD in kidneys ${ }^{23}$. In contrast, clinical acute and chronic GVHD developed in seven and four patients, respectively ${ }^{7}$. Some patients exhibited tubular atrophy, PTC loss, and interstitial fibrosis, resembling chronic rejection after kidney transplantation, suggesting development of chronic renal GVHD. In addition, C4d deposition in diffuse glomerular capillaries and patchy PTCs were noted in four patients. In this case series, renal TMA may have been involved in chronic renal GVHD after allogeneic HSCT. Moreover, some patients with $\mathrm{C} 4 \mathrm{~d}$ deposition might have had a humoral response in the kidney.

Renal TMA is not a common manifestation of renal GVHD. We attempted to use animal experiments to ex- 


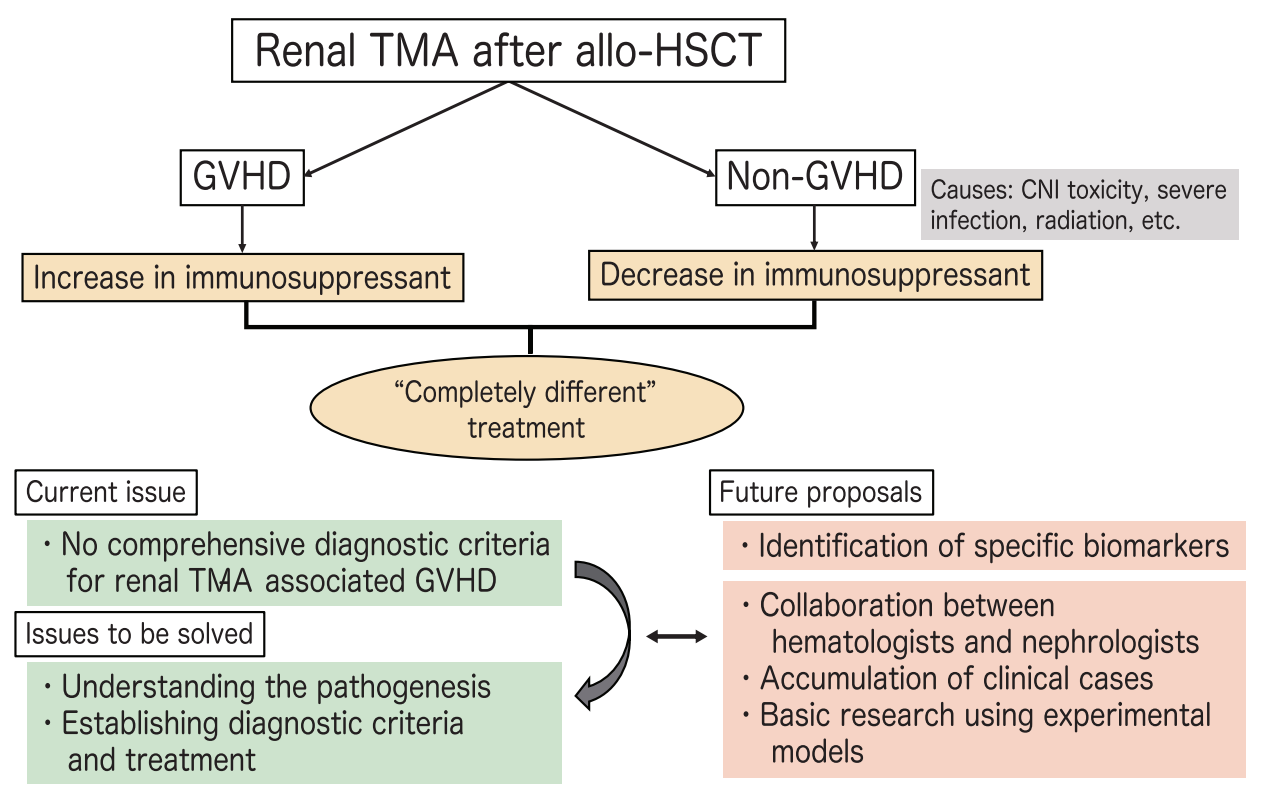

Fig. 3 Future perspectives for renal TMA-associated GVHD

The illustration shows current concerns, issues to be resolved, and our proposals.

amine the relationship between renal TMA and GVHD. We created a GVHD model of Lewis rats transplanted with Dark Agouti (DA) rat bone marrow ${ }^{34}$. Two to four weeks after transplantation, symptoms characteristic of severe GVHD were observed in skin, liver, and gastrointestinal tract. Simultaneously, PTC-itis, tubulitis, and glomerulitis accompanied by infiltration of donor $\mathrm{T}$ lymphocytes were confirmed in the kidneys, which strongly suggests acute renal GVHD. Another study using a murine model of acute GVHD in several tissues, including kidneys with capillaritis, endothelialitis, and tubulitis, reported findings suggesting renal GVHD ${ }^{35}$. However, these models did not yield clear evidence of severe endothelial cell injury in human studies. Therefore, it is necessary to further examine renal manifestation in the late stage of our model or to create and investigate another model of chronic GVHD.

If the cause of renal TMA is GVHD, kidney function might improve after resuming or increasing administration of immunosuppressive drugs. When we resumed or increased the dosage of prednisolone in three of four patients with renal TMA and C4d deposition diagnosed with renal GVHD, renal insufficiency improved (Fig. 2) ${ }^{8}$. This indicates that circulating antibodies to recipient endothelial cells are present and suggests that renal TMA can cause renal GVHD.

\section{Future Perspectives}

It has been common to reduce or stop immunosuppressive drugs when renal TMA develops after allogeneic
HSCT, because CNI and infectious diseases were believed to be causes of TMA. If, as suggested here, the cause of renal TMA is renal GVHD, conventional treatments would not be appropriate and it would be necessary to increase administration of immunosuppressive drugs. Evidence is increasing for the hypothesis that GVHD has a role in the development of renal TMA after allogeneic HSCT, but its pathology and treatment have not been clarified (Fig. 3). We will continue to identify clinicopathological features and specific biomarkers of renal TMA involved in GVHD by examining more cases and using animal experiment models. We hope to contribute to further characterizing the disease and determining its treatment.

Conflict of Interest: The authors declare no competing interests.

\section{References}

1. Niederwieser D, Baldomero H, Szer J, et al. Hematopoietic stem cell transplantation activity worldwide in 2012 and a SWOT analysis of the Worldwide Network for Blood and Marrow Transplantation Group including the global survey. Bone Marrow Transpl. 2016;51:1-8. doi: $10.1038 / \mathrm{bmt} .2016 .18$

2. Ando M. An overview of kidney disease following hematopoietic cell transplantation. Intern Med. 2018;57:1503-8. doi: 10.2169/internalmedicine.9838-17

3. Ando $\mathrm{M}$, Ohashi $\mathrm{K}$, Akiyama $\mathrm{H}$, et al. Chronic kidney disease in long-term survivors of myeloablative allogeneic haematopoietic cell transplantation: prevalence and risk factors. Nephrol Dial Transplant. 2010;25:278-82. doi: $10.1093 / \mathrm{ndt} / \mathrm{gfp} 485$

4. Hingorani SR. Renal complications of hematopoietic cell transplantation. N Engl J Med. 2016;374:2256-67. 
5. Shingai N, Morito T, Najima Y, et al. Early-onset acute kidney injury is a poor prognostic sign for allogeneic SCT recipients. Bone Marrow Transpl. 2015;50:1557-62. doi: 10.1038/bmt.2015.188

6. Mii A, Shimizu A, Masuda Y, et al. Renal thrombotic microangiopathy associated with chronic humoral graft versus host disease after hematopoietic stem cell transplantation. Pathol Int. 2011;61:34-41. doi: 10.1111/j.14401827.2010.02608.x

7. Mii A, Shimizu A, Kaneko T, et al. Renal thrombotic microangiopathy associated with chronic graft-versus-host disease after allogeneic hematopoietic stem cell transplantation. Pathol Int. 2011;61:518-27. doi: 10.1111/j.14401827.2011.02704.x

8. Mii A, Shimizu A, Kaneko T, Nakayama K, Yamaguchi H, Tsuruoka S. Renal thrombotic microangiopathy after hematopoietic stem cell transplantation: involvement of chronic graft-versus-host disease. Kidney Int Reports. 2018;3:743-7. doi: 10.1016/j.ekir.2017.12.013

9. Changsirikulchai S, Myerson D, Guthrie KA, Mcdonald GB, Alpers CE, Hingorani SR. Renal thrombotic microangiopathy after hematopoietic cell transplant: role of GVHD in pathogenesis. Clin J Am Soc Nephrol. 2009;4: 345-53. doi: 10.2215/CJN.02070508

10. Lopes JA, Jorge $S$, Silva $S$, et al. Acute renal failure following myeloablative autologous and allogeneic hematopoietic cell transplantation. Bone Marrow Transpl. 2006; 38:707. doi: 10.1038/sj.bmt.1705513

11. Ando M, Mori J, Ohashi K, et al. A comparative assessment of the RIFLE, AKIN and conventional criteria for acute kidney injury after hematopoietic SCT. Bone Marrow Transpl. 2010;45:1427-34. doi: 10.1038/bmt.2009.377

12. Ellis MJ, Parikh CR, Inrig JK, Kanbay M, Patel UD. Chronic kidney disease after hematopoietic cell transplantation: a systematic review. Am J Transpl. 2008;8:2378-90. doi: 10.1111/j.1600-6143.2008.02408.x

13. Shlipak MG, Fried LF, Cushman M, et al. Cardiovascular mortality risk in chronic kidney disease comparison of traditional and novel risk factors. J Amer Med Assoc. 2005;293:1737-45.

14. Webster AC, Nagler EV, Morton RL, Masson P. Chronic kidney disease. Lancet. 2017;389:1238-52. doi: 10.1016/S 0140-6736(16)32064-5

15. Jo T, Arai Y, Kondo T, et al. Biology of blood and marrow transplantation chronic kidney disease in long-term survivors after allogeneic hematopoietic stem cell transplantation: retrospective analysis at a single institute. Biol Blood Marrow Transpl. 2017;23:2159-65. doi: 10.1016/j. bbmt.2017.08.016

16. Hingorani SR, Seidel K, Lindner A, Aneja T, Schoch G, Mcdonald G. Albuminuria in hematopoietic cell transplantation patients: prevalence, clinical associations, and impact on survival. Biol Blood Marrow Transpl. 2008;14: 1365-72. doi: 10.1016/j.bbmt.2008.09.015

17. Hingorani S, Pao E, Stevenson $\mathrm{P}$, et al. Changes in glomerular filtration rate and impact on long-term survival among adults after hematopoietic cell transplantation a prospective cohort study. Clin J Am Soc Nephrol. 2018;13:866-73. doi: 10.2215/CJN.10630917

18. Luo XD, Liu QF, Zhang Y, et al. Nephrotic syndrome after allogeneic hematopoietic stem cell transplantation: etiology and pathogenesis. Blood Cells Mol Dis. 2011;46: 182-7.

19. Byrne-Dugan CJ, Collins AB, Lam AQ, Batal I. Membranous nephropathy as a manifestation of graft-versus-host disease: association with HLA antigen typing, phospholipase A2 receptor, and C4d. Am J Kidney Dis. 2014;64: 987-93.

20. Rao S. Nephrotic syndrome in patients with peripheral blood stem cell transplant. Am J Kidney Dis. 2005;45:7805. doi: 10.1053/j.ajkd.2005.01.003
21. Brukamp K, Doyle AM, Bloom RD, Bunin N, Tomaszewski JE, Cizman B. Nephrotic syndrome after hematopoietic cell transplantation: do glomerular lesions represent renal graft-versus-host disease? Clin J Am Soc Nephrol. 2006;1:685-94. doi: 10.2215/CJN.00380705

22. Seconi J, Watt V, Ritchie DS. Nephrotic syndrome following allogeneic stem cell transplantation associated with increased production of TNF-alpha and interferon-gamma by donor T cells. Bone Marrow Transplant. 2003;32:44750. doi: $10.1038 /$ sj.bmt.1704151

23. Kusumi E, Kami M, Hara S, et al. Postmortem examination of the kidney in allogeneic hematopoietic stem cell transplantation recipients: Possible involvement of graftversus-host disease. Int J Hematol. 2008;87:225-30. doi: 10.1007/s12185-008-0026-2

24. Ferrara JLM, Levine JE, Reddy P, Holler E. Graft-versushost disease. Lancet. 2009;373:1550-61. doi: 10.1016/S 0140-6736(09)60237-3

25. Cooke KR, Luznik L, Sarantopoulos S, et al. The biology of chronic graft-versus-host disease: a task force report from the national institutes of health consensus development project on criteria for clinical trials in chronic graftversus-host disease. Biol Blood Marrow Transplant. 2017; 23:211-34. doi: 10.1016/j.bbmt.2016.09.023

26. Kanamori H, Maruta A, Sasaki S, et al. Diagnostic value of hemostatic parameters in bone marrow transplantassociated thrombotic microangiopathy. Bone Marrow Transplant. 1998;21:705-9. doi: 10.1038/sj.bmt.1701151

27. Matsuda Y, Hara J, Osugi Y, et al. Serum levels of soluble adhesion molecules in stem cell transplantation-related complications. Bone Marrow Transplant. 2001;27:977-82. doi: $10.1038 /$ sj.bmt.1703026

28. Biedermann BC, Sahner S, Gregor M, et al. Mechanisms of disease endothelial injury mediated by cytotoxic $\mathrm{T}$ lymphocytes and loss of microvessels in chronic graft versus host disease. Lancet. 2002;359:2078-83.

29. Biedermann BC. Vascular endothelium and graft-versushost disease. Best Pract Res Clin Haematol. 2008;21:12938. doi: 10.1016/j.beha.2008.02.003

30. Williams WW, Tolkoff-rubin N, Colvin RB. Clinical role of the renal transplant biopsy. Nat Rev Nephrol. 2013;8:11021. doi: 10.1038/nrneph.2011.213.Clinical

31. Jodele S, Laskin BL, Dandoy CE, et al. A new paradigm: Diagnosis and management of HSCT-associated thrombotic microangiopathy as multi-system endothelial injury. Blood Rev. 2015;29:191-204. doi: 10.1016/j.blre.2014.11.001

32. Siami K, Kojouri K, Swisher KK, Selby GB, George JN, Laszik ZG. Thrombotic Microangiopathy after allogeneic hematopoietic stem cell transplantation: an autopsy study. Transplantation. $2008 ; 85: 22-8$. doi : 10.1097 / 01 . tp.0000297998.33418.7e

33. Jodele S, Fukuda T, Vinks A, et al. Eculizumab therapy in children with severe hematopoietic stem cell transplantation e associated thrombotic microangiopathy. Biol Blood Marrow Transplant. 2014;20:518-25. doi: 10.1016/j. bbmt.2013.12.565

34. Higo S, Shimizu A, Masuda Y, et al. Acute graft-versushost disease of the kidney in allogeneic rat bone marrow transplantation. PLoS One. 2014;9:e115399.

35. Niculescu F, Niculescu T, Nguyen P, et al. Both apoptosis and complement membrane attack complex deposition are major features of murine acute graft-vs.-host disease. Exp Mol Pathol. 2005;79:136-45. doi: 10.1016/j. yexmp.2005.03.007

(Received, February 10, 2019)

(Accepted, August 23, 2019)

(J-STAGE Advance Publication, November 28, 2019) 